\title{
Pulmonary alveolar proteinosis secondary to chronic chlorine occupational inhalation
}

\begin{abstract}
We present a 38-year-old patient with a history of two months of dyspnea mMRC IIIII, cough, low-grade fever and impregnation syndrome. Clinical and radiologic studies suggested a diagnosis of Pulmonary Alveolar Proteinosis (PAP) confirmed by transbronchial biopsy (TBB) and bronchoalveolar lavage (BAL). PAP is one of the "orphan diseases" of the lung, with very low prevalence and incidence. Three clinical variants are known: Idiopathic, Acquired and Secondary. Due to the nature of our patient's work setting, we diagnosed occupational PAP secondary to the chronic inhalation of chlorine in a poorly ventilated work environment as well as secondary to inadequate respiratory protection. The patient received one session of Total Pulmonary Lavage (WLL) and he clinically responded and improved.
\end{abstract}

Keyword: pulmonary, transbronchial biopsy, pulmonary lavage, chronic chlorine, pulmonary alveolar proteinosis
Volume 5 Issue 3 - 2018

Darío Raúl Rey,' José Antonio González²

'Honorary Consultant Pneumology, Hospital general De Agudos Dr. ETornú, Argentina

${ }^{2}$ Chief Division Pneumology, Hospital general De Agudos Dr. E Tornú, Argentina

Correspondence: Darío Raúl Rey, Honorary Consultant Pneumology, Hospital general De Agudos Dr. ETornú, Buenos Aires,Argentina, Email darioraul.rey@gmail.com

Received: May 14, 2018 | Published: June 19, 2018

\section{Introduction}

Pulmonary Alveolar Proteinosis (PAP) is one of the "orphan diseases" of the lung, whose peculiarity consists in an intra-alveolar accumulation of a lipo-proteinaceous material related to surfactant, which interferes with gas exchange, presenting with symptoms and signs varying in intensity. Initially described by Rosen, Castleman and Liebow in 1958, its frequency is very low: both Diksen and Ben Dov estimate an incidence in 0.37 cases $/ 100,000$ people and prevalence in 3.7/1,000,000 inhabitants. ${ }^{1-3}$

Three clinical variants are known: Idiopathic, ${ }^{4}$ Acquired (or autoimmune) and Secondary, which occur during bacterial infections, HIV, hematological malignancies, inhalation of fumes, organic or inorganic particles, such as cotton or aluminum respectively. ${ }^{5-14}$ The diagnosis of PAP secondary to chronic chlorine occupational inhalation is even more unusual and rare, warranting this case report.

\section{Case report}

Our subject is a 38 years old male with a history of 40 pack-years of tobacco use, as well as alcoholism. He has a history of hypertension. He has been employed as a tannery worker for the last 8 years and he presented with a 2-month history of mMRC II-III associated cough, fevers, night sweats and impregnation syndrome. He was referred to a regional hospital. In the Emergency Room, he was found to have hypoxemia associated with bilateral nodule-like opacities on his Chest X-ray and was admitted. On clinical examination, he was sub-cyanotic with an Oxygen saturation at rest breathing air of $86 \%$. He was found to have "Clubbed" fingers, pulse rate of 88 per minute, blood pressure of $110 / 60 \mathrm{~mm} \mathrm{Hg}$. Respiratory examination revealed fine moist rales in middle and lower fields of both lungs.

Studies carried out: Serology for HIV, B and C hepatitis: Negative.

Chest X-ray (CXR): Images with alveolar pattern appearance on both pulmonary fields with predominance of middle and lower zones (Figure 1).

A Chest Computed tomography scan of the thorax (CT): showed no mediastinal adenopathies. Extensive alveolar commitment of bilateral and geographic distribution at the level of both lungs with a "crazy paving" type. Ventilated areas at the level of apical and posterior segments of right superior lobe (Figure 2).

Spirometry: revealed FVC 1380ml (31\%) FEV1 1240ml (35\%) and FEV1/ FVC $89 \%$ corresponding to a Severe Restrictive Pattern.

Due to a lack of access to advanced pulmonary testing facilities at the treating hospital, neither a DLCO nor titration of Antibodies Anti-Granulocyte-
Macrophage Colony-Stimulating Factor (anti GM-CSF), in blood were made. Test performed included:

i. A fiberoptic bronchoscopy (FOB): "No specific lesions up to third orderbronchi, showing no signs of hemorrhage or alteration of the pulmonary architecture"

ii. A transbronchial biopsy (TBB) whose histopathology showed "alveolar spaces and respiratory bronchioles filled with eosinophilic material PASpositive characteristic of PAP" (Figure 3).

iii. A Bronchoveolar lavage (BAL) was performed and sent for bacteriological and mycological studies (negative results).

iv. A Cytological study of BAL showed "macrophages with amorphous acidophilic material in cytoplasm and as background material with diffuse distribution and in small accumulations. Positive for PAS technique. Negative for neoplastic cells".

v. Abdominal and urogenital ultrasound: no particularities.

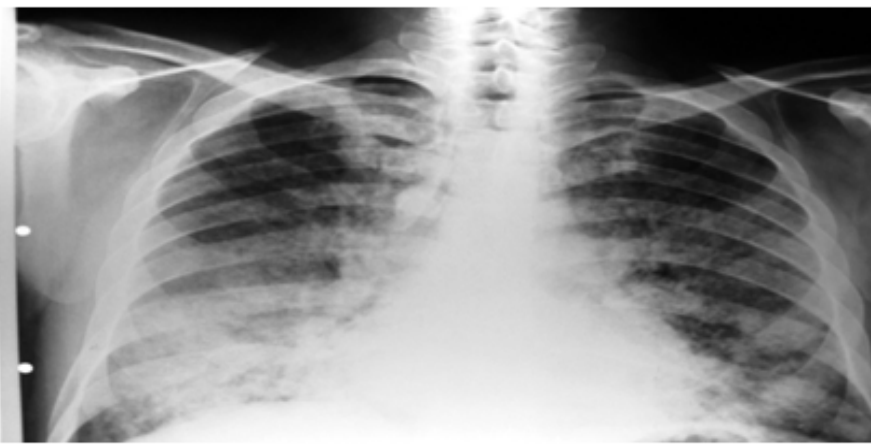

Figure I Alveolar pattern appearance on both pulmonary fields with predominance of middle and lower zones.

The patient gave a more detailed history of his work-related activities over the period of 8 years in tanning, which consisted of immersing raw leathers in a solution with hydrochloric acid, leaving them drying for 2-3 months prior to processing leathers. He performed these work tasks with inadequate respiratory protection on broad, uncovered patches, despite which, he usually presented symptoms of irritation in the upper airways and ocular apparatus (irritative cough and conjunctival inflammation) during and after work. Based on the studies carried out as well as his work history, the case was interpreted as a Secondary Alveolar Proteinosis due to chronic chlorine exposure. 


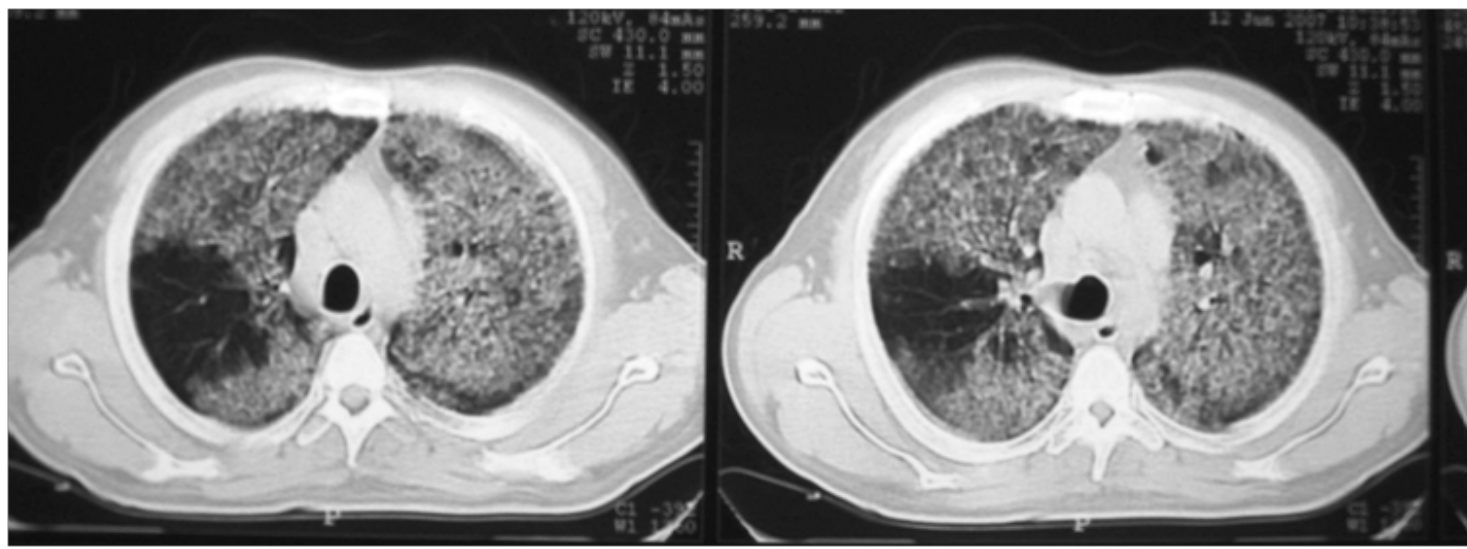

Figure 2 Extensive alveolar commitment of bilateral and geographic distribution at the level of both lungs with a crazy paving type.

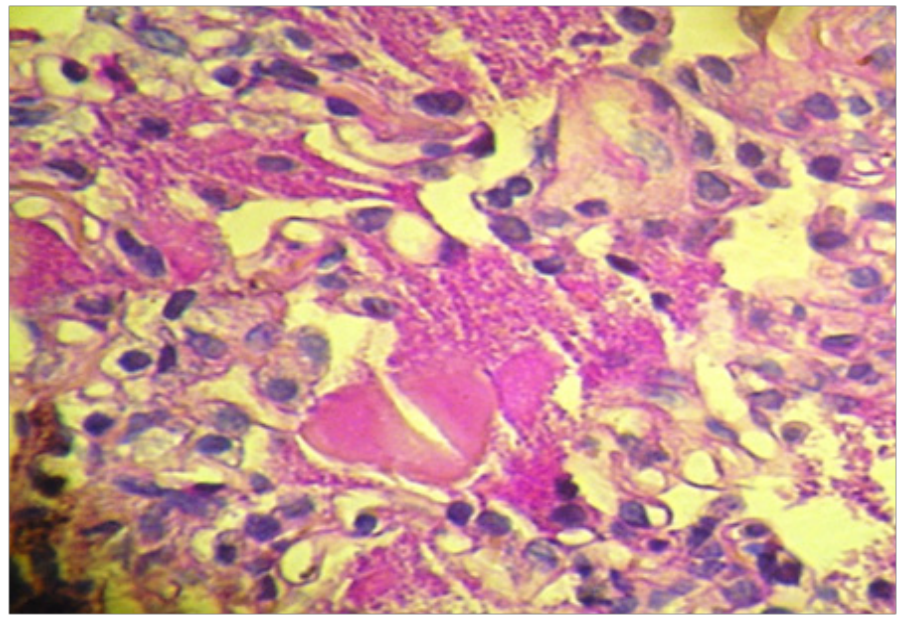

Figure 3 Alveolar Proteinosis PAS Technique.

Treatment Intervention: One session of whole lung lavage (WLL) was performed, evolving favorably and improving oxygenation without requiring supplemental oxygen. Laboratory at discharge: GR $6.380 .000 / \mathrm{mm} 3$ Hto $53 \%$ GB $14.900 / \mathrm{mm} 3$ Oxygen saturation at rest breathing air $93 \%$. Normal Ionogram. It was decided to be discharged from hospital and periodically examined on External Office, but the patient did not return to control.

\section{Discussion}

Accidental, repeated and chronic chlorine inhalation at low environmental concentrations in the industry, causes irritation of the oropharyngeal and ocular mucous membranes, chronic irritant cough and an influenza-like condition. ${ }^{15}$ These episodes cause a limitation to airflow and changes in lung function, facts that have been well researched in the specialized literature. ${ }^{16}$

The surface of the pulmonary alveolus is covered by a thin line of surfactant, composed of $90-95 \%$ of lipids and $5-10 \%$ of proteins, which reduces surface tension; it prevents collapse and transudation of capillary fluid in the alveolar lumen. The surfactant proteins (A-B-C and D) have properties such as partaking in metabolism, opsonizing pathogenic microorganisms and stimulating the functional capacity of alveolar macrophages. ${ }^{17}$

In PAP, fundamental finding consists of a defect of the alveolar macrophages to process the pulmonary surfactant, which accumulates in the intra-alveolar amorphous material as well as in the detritus of these cells. Medical knowledge about PAP was intensified when studying homozygous mice, with suppression of the gene producing GM-CSF, developed a PAPlike disease. The administration of aerosolized GM-CSF to the affected mice, reversed the lung lesions. ${ }^{18}$

PAP can present 3 clinical variants:

i. Congenital (exceptional), which is transmitted in a recessive autonomic manner caused by a genetic mutation that causes a deficiency in protein B surfactant.

ii. The Acquired or Autoimmune cause of unknown origin, in which there is no family predisposition or apparent factors responsible for its appearance.

iii. The Secondary one, caused by infectious, occupational or hematological causes

As in Sarcoidosis, the clinical course in these patients can be variable and non-specific and present a clinical dissociation in relation to what is seen on CXR and by testing. Progressive dyspnea and unproductive cough usually have an evolution of 6-7 months in $75 \%$ of the cases and the clinical findings show inspiratory rales in $50 \%$, cyanosis in $25 \%$ and in a very low percentage, the presence of "clubbing fingers". Weight loss and low-grade fever are not as frequent. In $13 \%$ of cases, a respiratory infection can be associated with Nocardiaasteroides. ${ }^{19}$

The diagnostic studies identified full alveolar compromise located in the middle and lower fields, with predominance in the perihilar regions, which configures a "butterfly wing"- like. ${ }^{20}$ However, it is CT that provides high diagnostic yield by confirming the existence of alveolar geographic consolidation, with or without air bronchogram, which is associated with "ground glass opacitiy" and thickening of the interlobular and septal septa, giving the appearance known as "crazy paving". Although it is not specific to PAP, as in this case, it may suggest the diagnostic orientation associated with a clinical and functional context..$^{21,22}$ Functional evaluation of these patients reveals hypoxemia, a restrictive respiratory pattern, a mild decrease in FVC and TLC, as well as a severe decrease in DLCO, directly related to the involvement of the lung parenchyma.

Although the clinical picture as CT suggests the diagnosis, high suspicion is presented before performing a FOB, since the washing liquid presents an opalescent and milky appearance that, on microscopic examination, has foamy macrophages as well as eosinophilic bodies in a background of granular material that is stained with PAS. LDH is elevated in both serum and bronchial lavage..$^{20}$ Using electron microscopy, amorphous granular waste with osmophilic structures like the so-called lamellar bodies can be observed. 
The "gold standard" is the lung biopsy that allows us to appreciate the histological pattern described in our observation, although performing a transbronchial biopsy also has a high yield because it is a diffuse pulmonary disease. ${ }^{23}$ An interesting fact linked to PAP, is that anti-GM-CSF antibodies were detected in the BAL practiced in the acquired forms, which are found neither in the other variants of the PAP, nor in other pulmonary conditions and, by logic, neither in the normal lung. This finding has allowed the development of a serological test that has a sensitivity of $100 \%$ and a specificity of $98 \%$ for the diagnosis of the acquired variant of PAP ${ }^{24}$ Titration of high GM-CSF, correlates with a lower possibility of therapeutic response..$^{25}$ The therapeutic behavior to be carried out in this infrequent condition depends on its clinical form. In Congenital, the performance of a pulmonary transplant, successfully allowed the restitution of lung function in patients with PAP due to lack of surfactant B protein. ${ }^{24}$

In Secondary PAP, unilateral total pulmonary lavage (WLL) is performed. This is a traditional physical method that has given good results. Seymour reports that $63 \%$ of PAP cases are submitted to WLL, calculating that $20 \%$ of them remain free of symptoms after 3 years. It is as safe to perform as bilatera washing, keeping patients free of symptoms for an average of 15 months. This author found a survival of $94 \%$ of patients undergoing WLL versus $85 \%$ of those who did not undergo this procedure. ${ }^{19}$ In localized clinical forms of PAP, Cheng advocates the use of FOB with good results. ${ }^{26}$ Complications that can occur (hypoxemia, pneumonia, distress and pneumothorax) are scarce. ${ }^{19}$

The WLL is an unregulated procedure and with which the different researchers act with success, although without a non-uniform methodology. Trying to establish such behavior, Campo et al. sent a questionnaire to 79 Centers about the technique used, lung initially chosen, amount of physiological solution used, etc. answering 20 Centers from 14 countries. ${ }^{27}$ The summary of the results obtained was as follows:

a. It is done under general anesthesia, with double-lumen endobronchial tube in two sessions with 1-2 weeks interval.

b. The instilled solution is physiological at $37^{\circ} \mathrm{C}$

c. The aliquot of mean serum was $800 \mathrm{ml}$ and the total amount in the procedure was 5.4 liters per lung.

d. $50 \%$ of Centers initially chose the left lung, while the remaining one did so based on the radiographic evidence.

e. WLL is a non-regulated procedure and with which the different researchers act with success, although without a non-uniform methodology.

In the Acquired or autoimmune clinical variant, where the presence of antiGM-CSF antibodies is detected, therapeutic successes have been reported both with the administration of GM-CSF subcutaneously and by an aerosolized form. This therapy is well tolerated and results have been good in both modes of administration, although cohorts have been small in number. The optimum dose must still be adjusted and the knowledge that its application can cause distant effects..$^{25,28-30}$

\section{Conclusion}

PAP is an "orphan disease" whose natural evolution is not completely known. Its pathogenesis has been partially clarified, especially in the acquired or autoimmune form, by the implication of GM-CSF and the presence of antiGM-CSF antibodies. The published casuistry of around 500 cases according to Ioachimescu, shows both patients with remission and spontaneous remission as with prolonged lapses of quiescence..$^{31}$ Beccaria in her series of 21 patients undergoing WLL, only had 30\% relapses although they persisted with some degree of functional impairment. ${ }^{32}$ With this therapeutic modality according to Pressneill, the survival at 5 years is calculated at $80 \%$. $^{33-34}$

\section{Acknowledgments}

None.

\section{Conflict of interest}

Author declares there is no conflict of interest.

\section{References}

1. Rosen S, Castleman B, Liebow A. Pulmonary alveolar proteinosis. $N$ Engl J Med. 1958;258(23):1123-1142.

2. Ben Dov I, Kishinevsky Y, Roznman J, et al. Pulmonary alveolar proteinosis in Israel: ethnics clusterings. Isr Med Assoc J. 1999;1(2):75-78.

3. Dirksen U, Nishinakamura R, Groneck P, et al. Human pulmonary alveolar proteinosis associated with a defect in GM-CSF/IL3-5 receptor common beta chain expression. J Clin Invest. 1997;100(9):2211-2217.

4. Teja K, Cooper P, Squires J, et al. Pulmonary alveolar proteinosis in four siblings. N Engl J Med. 1981;305(23):1390-1392.

5. Gacouin A, Le Tulzo Y, Suprin E, et al. Acute respiratory failure caused by secondary alveolar proteinosis in a patient with acute myeloid leukemia: a case report. Intensive Care Med. 1998;24(3):265-267.

6. Aymard J, Gyger M, Lavallee R, et al. A case of pulmonary alveolar proteinosis complicating chronic myelogenous leukemia. A peculiar pathologic aspect of busulfanlung? Cancer. 1984;53(5):954-956.

7. Dawkins S, Gerhard H, Nevin M. Pulmonary alveolar proteinosis: a possible sequel of N02 exposure. J Occup Med. 1991;33(5):638-641.

8. Keller C, Frost A, Cagle P, et al. Pulmonary alveolar proteinosis in a painter with elevated pulmonary concentrations of titanium. Chest. 1995;108(1):277-280.

9. Orriols R, Ferrer J, Tura J, et al. Sicca syndrome and silicoproteinosis in a dental technician. Eur Resp J. 1997;10(3):731-734.

10. Sauni R, Järvenpää R, Livonen E, et al. Pulmonary alveolar proteinosis induce by silica dust? Occup Med. 2007;57(3):221-224.

11. Bakhos R, Gattuso P, Arcot C, et al. Pulmonary alveolar proteinosis: an unusual association with Mycobacterium avium-intracellulare infection and lymphocitic interstitial pneumonia. South Med J. 1996;89(8):801-802.

12. Ruben F, Talamo T. Secondary pulmonary alveolar proteinosis occurring in two patients with Acquired Immunodeficiency Syndrome. Am J Med. 1986;80(6):1187-1190.

13. Thind G. Acute pulmonary alveolar proteinosis due to exposure to cotton dust. lung India. 2009;26(4):152-154.

14. Chew R, Nicam S, Sivakumaran P. Alveolar proteinosis associated with aluminium dust inhalation. Occup Med. 2016;66(6):492-494.

15. Courteau J, Cushman R, Bouchard F, et al. Survey of construction workers repeatedly exposed to chlorine over a three to six month period in a pulpmill : Exposure and symptomatology. Occup Environm Med. 1994;51(4):219-224

16. Mehta A, Henneberger P, Torén K, et al. Airflow limitation and changes in pulmonary function among bleachery workers. Eur Resp J. 2005;26(1):133-139.

17. Weaver T, Whitsett J. Function and regulation of expression of pulmonary surfactant-associated proteins. Biochem J. 1991;273(2):249-264.

18. Stanley E, Lieschke G, Grail D, et al. Granulocyte/macrophage colonystimulating factor-deficient mice show no major perturbation of hematopoiesis but develop a characteristic pulmonary pathology. Proc Nat AcadSci USA. 1994;91(12):5592-5596.

19. Seymour J, Presneill J. Pulmonary alveolar proteinosis: progress in the first 44 years. Am J Respir Crit Care Med. 2002;166(2):215-235. 
20. Wang E, Stern E, Schmidt R, et al. Diagnosing pulmonary alveolar proteinosis: a review and update. Chest. 1997;111(2):460-466.

21. Rossi S, Erasmus J, Volpacchio M, et al. Crazy paving pattern at thin section CT of the lungs: Radiologic-pathologic overview. Radiographics. 2003;23(6):1509-1519.

22. Holbert J, Costello P, Li W, et al. CT features: of pulmonary alveolar proteinosis. AJR. 2001;176(5):1287-1294.

23. Leslie K. A pathologic approach to the classification of Idiopathic Interstitial Pneumonias. Chest. 2005;128(5 Suppl 1):S513-519.

24. Kitamura T, Uchida K, Tanaka N, et al. Serological diagnosis of idiopathic pulmonary alveolar proteinosis. Amer J Resp Crit Care Med. 2000;162(2 Pt 1):658-662.

25. Venkateshiah B, Yan T, Bonfield T, et al. An open-label trial of GM-CSF therapy for moderate symptomatic pulmonary alveolar proteinosis. Chest. 2006;130(1):227-237.

26. Cheng S, Chang H, Lau H, et al. Pulmonary alveolar proteinosis: Treatment by bronchofiberoptic lobar lavage. Chest. 2002;122(4):1480-1485.

27. Campo I, Luisetti M, Griese M, et al. Whole lung lavage therapy for pulmonary alveolar proteinosis: A global survey of current practices and procedures. Orphanet J Lung Dis. 2016;11(1):115-124.
28. Barraclough R, Gillies A. Pulmonary alveolar proteinosis: a complete response to GM-CSF therapy. Thorax. 2001;56(8):664-665.

29. de Vega M, Sanchez-Palencia A, Ramirez A, et al. GM-CSF therapy in pulmonary alveolar proteinosis. Thorax. 2002;57(9):837.

30. Wylam M, Ten R, Prakash H, et al. Aerosol granulocyte-macrophage colony-stimulating factor for pulmonary alveolar proteinosis. Eur Resp J. 2006;27(3):585-593.

31. Ioachimescu O, Kavuru M. Pulmonary alveolar proteinosis. Chron Resp Dis. 2006;3(3):149-159.

32. Beccaria M, Luisetti M; Rodi G et al. Long-term durable benefit after whole lung lavage in pulmonary alveolar proteinosis. Eur Resp J. 2004;23(4):526-531.

33. Presneill J, Nakata K, Incue Y, et al. Pulmonary alveolar proteinosis. Clin Chest Med. 2004;25(3):593-613.

34. Hamvas A, Nogee 1, Mallory G, et al. Lung transplantation for treatment of infants with surfactant protein B deficiency. $J$ Pediatr. 1997;130(2):231-239. 